\title{
Responsabilidad civil médica y contrato de seguro: Sobre las cláusulas de delimitación temporal
}

\section{Alfredo Ferrante ${ }^{1,2}$}

1. Profesor. Universidad Alberto Hurtado. Santiago, Chile.

2. Doctor en Derecho, con mención europea, por la Universidad de Oviedo. Oviedo, España.

\section{Medical Liability and insurance contract: About claims made clause.}

\begin{abstract}
The purpose of this paper is to present a particular type of clause that can be found in an insurance contract related to professional activities: the claims made clause. This clause presents multiple legal problems which have a direct impact on the practical application in the medical liability. One of these occurs in cases where the damage is not covered by the insurance company even if the policy has been properly paid by the insured, and the compensation must be paid by the doctor to the victim.
\end{abstract}

Key words: Medical liability, claims made, insurance contract.

Resumen. El presente artículo tiene por objeto dar a conocer un particular tipo de cláusula que puede encontrarse en las pólizas de seguro por actividades profesionales: la cláusula de delimitación temporal llamada claims made. Ésta presenta múltiples problemáticas desde el punto de vista jurídico, que tienen directa incidencia en el ámbito práctico de la responsabilidad médica. Una de ellas, se produce en aqueIlos casos en que los siniestros no reciben cobertura de la compañía, aun estando vigente y debidamente pagada la póliza por el asegurado, debiendo asumirse el pago de la indemnización por el propio médico frente a la víctima.

Palabras claves: Responsabilidad civil médica, cláusula de delimitación temporal, cláusula claims made, contrato de seguro.

Ferrante A. Responsabilidad civil médica y contrato de seguro: sobre las cláusulas de delimitación temporal. Rev Chil Radiol 2018; 24(2): 48-54.

Correspondencia: Alfredo Ferrante / aferrante@uahurtado.cl

Trabajo enviado el 03 de junio de 2018. Aceptado para publicación el 26 de julio de 2018.

\section{Introducción}

La responsabilidad civil derivada de la actividad médica ${ }^{1,2,3}$ ha concitado un incesante estudio en Chile desde la masificación de las demandas indemnizatorias a fines de los años 80 , siendo hoy aceptada, incluso, la procedencia de obligaciones de resultadoi. Lo anterior, muestra que las indemnizaciones pueden también derivar de la obtención de un determinado logro, con independencia de que sea una actividad aislada ${ }^{4,5,6,7}$ o en equipo ${ }^{6,7}$, en un ámbito en que naturalmente se consideró la presencia de obligaciones de diligencia.

Un mecanismo que atenúa los efectos patrimoniales es la contratación de un seguro de responsabilidad civil profesional. Aunque en Chile no exista la obligación de suscribirlo -como en otras legislaciones (v.gr. Italia) - casi la totalidad de los médicos opta por contratarlo, siendo además una exigencia efectiva, pues, a menudo, las clínicas no permiten que un profesional sin seguro opere en sus instalaciones.

El objetivo de este artículo, es evidenciar algunos aspectos prácticos y específicos de estos contratos, en particular, cuando se incorporan las llamadas cláusulas claims made, que delimita temporalmente la responsabilidad. Ellas, sólo abordadas en parte por la doctrina ${ }^{8,9,10,11}$ producirán, en determinados casos, un efecto placebo, por cuanto pueden excluir la protección del seguro y consecuentemente, obligar al médico a asumir la totalidad la indemnización reclamada. En dichas situaciones, a pesar de haberse contratado el seguro profesional correspondiente y se hayan pagado las primas, la póliza podría no cubrir la reparación del daño.

Con la finalidad de orientar al médico a celebrar 
su póliza, prospectaré dos modelos que actualmente pueden contratarse en Chile: el sistema tradicional, fiel a la impostación clásica del contrato de seguro y el sistema con cláusulas claims made, que, en parte, lo deroga. Para analizar este aspecto se aportarán algunos de los modelos de pólizas depositados en la Superintendencia de Valores y Seguros.

\section{El punto de partida}

Usualmente los daños físicos son percibidos en el momento en que son provocados, por ejemplo, en un accidente de tránsito, una vez ocurrido el evento lesivo, simultáneamente podrá evidenciarse la producción del daño: de inmediato, o con bastante cercanía, se sabrá si hay algún lesionado al cual podría corresponder una indemnización por parte del conductor negligente o del propietario del vehículo.

En cambio, la peculiar naturaleza de los perjuicios derivados de una malpraxis médica justifica un análisis más detenido. En efecto, esta actividad profesional crea, a menudo, un desfase temporal entre el evento que ha dado lugar al daño (una cirugía, una equivocada diagnosis o una errada interpretación de una imagen ${ }^{12}$ ) y su manifestación. Piénsese en la omisión de un diagnóstico de cáncer, que se detecta tiempo después y que, posteriormente, conduce a la muerte del sujeto. Es decir, en algunos casos, el daño médico no se exterioriza inmediatamente, sino que tiene una manifestación tardía, por lo que surge la necesidad de contar con un seguro para paliar el riesgo de la malpraxis del profesional.

\section{Los elementos en juego}

Hechas estas premisas, para comprender la operatividad de este seguro, deben tenerse en cuenta ciertos elementos relacionados con posibles fechas distintas. La primera, es aquella en que se verifica el siniestro (evento dañoso); es decir, el momento en que el médico ha incurrido en una acción u omisión negligente. La segunda, es la solicitud de los daños y perjuicios, que será evacuada por la víctima o el asegurado. Finalmente, como tercer factor, entra en juego la fecha de validez de la póliza. Así, dependiendo de la línea temporal en que se mueven estos elementos, el médico, aunque se encuentre asegurado, podría quedar desprotegido si ha firmado una cláusula que libere a la compañía de toda indemnización. Esta situación se produciría dependiendo del modelo de seguro contratado, Efectivamente, pueden presentarse dos tipos de coberturas: el modelo tradicional (internacionalmente llamado por ocurrence) y otro modelo relacionado con cláusulas de delimitación temporal, denominadas claims made. Si el primero cubre el riesgo de la responsabilidad civil del asegurado por sus acciones $u$ omisiones, el segundo cubre el riesgo del daño patrimonial del asegurado, cuando se deniegan sus reclamaciones. Véanse a continuación los dos modelos que pueden delinearse.

\section{Primer modelo de cobertura:I hecho dañoso cometido dentro del periodo de validez de la póliza}

Este modelo de póliza de responsabilidad civil profesional tradicional transfiere, a la compañía, los riesgos dentro de su período de vigencia (cfr. arts. 512, 579 Código de Comercio, en adelante: C. Com), beneficiando al médico o tomador (institución hospitalaria o Colegio Médico), con la cobertura de la indemnización por daños a terceros. Sin detenerse aquí sobre los tipos de daños cubiertos, ni sobre el hecho de que se excluyen aquellos originados por dolo o culpa grave (cfr. art. 535 C.Com.), debe evidenciarse que, para este modelo, el siniestro es aquel que se verifica durante la validez de la póliza. De forma que incluso si los daños continuaran una vez expirados, tendrían cobertura (art. 532 C.Com.). porque, se abarcan aquellos generados una vez que se firma la póliza y mientras se encuentre vigente, alcanzando a los que hayan empezado con ésta y sigan manifestándose después.

Para este sistema, hay una limitación que excluye la procedencia del seguro. La primera, es que no se cubrirán daños generados antes de la firma y vigencia de la póliza. Este aspecto favorece al asegurado, pues lo protege, aunque la manifestación del daño y la reclamación se verifiquen fuera de la vigencia de la póliza. Lo anterior, resulta aún más efectivo considerando que estos seguros normalmente tienen duración anual. El modelo, en cambio, perjudica a la compañía aseguradora, no sólo porque se presume que el siniestro se produjo por un evento que hace responsable al asegurador (art. $531 \mathrm{C}$. Com), sino porque mantiene sus obligaciones incluso por un período -entre el final de la póliza y todo el tiempo de prescripción de posibles demandas- en que no se paga ninguna prima.

Un ejemplo aclarará la cuestión. Un médico posee una póliza de seguro con vigencia entre el 1/01/2017 $y$ el 1/01/2018 e incurre en una negligencia dentro de tal período. Aunque el paciente demande una indemnización luego del 1/01/2018, la póliza cubriría esta mala práctica, pues el daño se ha ocasionado durante la validez de la póliza, cuya cobertura se extiende hasta la prescripción de esta acción judicial. En este caso, es útil diferenciar el plazo de prescripción de las demandas de terceros con aquel que la ley de seguros establece para que los asegurados presenten reclamos a sus aseguradores. El primero varía entre 4 o 5 años dependiendo del tipo de responsabilidad alegada (extracontractual art. $2332 \mathrm{CC}^{\mathrm{ii}}$ o contractual art. 2514 y 2515 CC, respectivamente) y el segundo, 4 años (art. 541 CCom). Lo relevante de esta diferencia de plazos de prescripción es que, eventualmente, podría perjudicar al asegurado en el segundo modelo de póliza, como se verá (sub 
“¿Validez de las cláusulas claims made?”).

\section{El segundo modelo de referencia: la teoría del reclamo}

El modelo arriba mencionado comportaría muchas veces inconvenientes para el asegurador, en aquellos daños que pueden manifestarse con posterioridad al acto médico.

Para evitar esta excesiva cobertura, es que las partes pueden modificar -en parte- el contenido básico del contrato de seguro, mediante el principio de autonomía contractual. Considerando además que se trata de un "contrato de adhesión", es que las compañías aseguradoras han predispuesto modelos que compensen este sistema, incorporando las llamadas cláusulas claims made, es decir, un mecanismo de cobertura que depende no sólo del evento dañoso, sino (que, sobre todo) del momento en el cual se efectúe la demanda o reclamo (claim) de daños y perjuicios. Es este último factor el que marca el éxito del espectro asegurado y que se concreta mediante la incorporación de determinadas cláusulas que se refieren al reclamo efectuado (claims made, de ahí su nombre).

Existen varias tipologías de cláusulas claims made, sin embargo, esencialmente se presentan dos principales, las puras y las híbridas iii.

Una primera condición de aplicabilidad es que la reclamación se haya realizado durante el periodo de la póliza; no es necesario (a diferencia del modelo analizado en el epígrafe anterior), que el daño se haya producido una vez contratado el seguro. La cláusula claims made pura, cubre cualquier hecho producido antes del contrato, con una retroactividad ilimitada, mientras que la mixta restringe la retroactividad a una fecha que oscila entre 1, 3 o 5 años. Aquí la aseguradora inserta una cláusula de retroactividad (salvo, eventualmente, otros tipos, como la deeming o la sunset clause), impidiendo la indemnización de todo perjuicio provocado durante la validez de la póliza que haya sido reclamado después de su término.

Véanse dos ejemplos, retomando el anterior. Una cláusula pura, cubriría el daño si la víctima ha reclamado entre el 1/01/2017 y el 1/01/2018, con independencia de que la actividad médica se haya realizado en una fecha anterior al 1/01/2017, retroactivamente hasta el infinito. En el caso de una cláusula híbrida, la compañía cubriría el daño si: a) tanto la actividad médica como la reclamación se produjeron en el periodo comprendido entre el 1/01/2017 y el 1/01/2018 o b) se haya reclamado el daño durante la validez de la póliza, pero la negligencia se haya cometido antes de su firma (1/01/2017). Se aprecia así una cláusula de retroactividad con tiempo limitado (por ejemplo, un año atrás desde la validez de la póliza). En todos los demás casos (por ejemplo, el paciente reclama el 2/01/2018), el médico debería asumir la indemnización.

\section{Modelos a elegir en Chile: Pro y contra}

La ventaja de una póliza sujeta a cláusulas claims made podría darse por la extensión retroactiva, salvo para un profesional de nueva incorporación, quien empezará a ejercer con la celebración de su primer seguro. Esta circunstancia no justificaría el pago de una prima mayor ${ }^{8,9}$ respecto al sistema tradicional, aún más pensando que normalmente la póliza es anual.

No obstante, algunos problemas pueden plantearse. Por ejemplo, el caso de un médico que, a sabiendas de haber cometido un ilícito anterior, intente redactar una nueva póliza con eficacia retroactiva con altos maximales (en el caso de que la póliza anterior los tuviera más bajos). Dado que la buena fe se presume, será de cargo de la compañía de seguro demostrar la presencia de declaraciones mendaces, que excluirían la cobertura (cfr. arts. 524, 525, 535 y 539 C. Com). Otro aspecto importante, es compatibilizar la eventual superposición de pólizas contratadas con distintas compañías, que se rijan por los dos modelos: aunque ambas cubrirán el supuesto dañoso, pasar del primero al segundo, sería beneficioso (salvo la diferencia de maximales); pero no valdría el razonamiento en el caso contrario, ya que el sujeto quedaría descubierto en el caso de manifestación tardía de un daño producido antes de la celebración de la segunda póliza. En este sentido, el haber contratado inicialmente una clase de póliza claims made, produce casi una automática y necesaria contratación de otra de igual tipología. Esto puede contribuir a retener al contratante con la misma compañía aseguradora, falseando el regular desarrollo de la competencia perfecta.

Ahora bien, si el fundamento de este contrato es la tutela de terceros, debería reprocharse un mecanismo que limite la cobertura aseguradora, más si se piensa que se está creando un modelo asegurador entre contratantes que, sin embargo, depende de un factor externo: la demanda del tercero, quien desconoce la importancia del momento en el que debe presentarla. ¿Valdría una reclamación preventiva por parte del médico, o se necesitaría exclusivamente la demanda de la víctima para la aplicación de la cláusula y la correspondiente medida aseguradora? Siendo que la reclamación se condiciona a que sea realizada por el tercero, es difícil reprochar al contratante el no haber realizado el reclamo durante de la vigencia de la póliza, ya sea que se trate de un profesional, o un consumidor común.

\section{¿Validez de las cláusulas claims made?}

En Chile la Superintendencia de Valores y Seguros predispone un mecanismo preventivo de depósito de pólizas que controla las condiciones generales de las mismas. Sin embargo, al haberse abandonado la vigilancia efectiva que originariamente ésta realizaba mediante un informe legal, en la actualidad el depósito de la póliza no conlleva ninguna garantía 
de que los textos cumplan con las normas legales y reglamentarias ${ }^{14}$. Por eso es necesario preguntarse si jurídicamente, es legítima una cláusula claims made, ya que, de hecho, existen argumentos que podrían justificar su invalidez.

Primeramente, debe asumirse que el riesgo es un elemento imprescindible del contrato de seguro (cfr. arts. 512, 513 letra t), art. 521 C. Com). Entonces, cabe preguntarse si la existencia de un riesgo putativo puede garantizar la validez de una cláusula claims made, como sería asegurar un evento retroactivo, previo la celebración de la póliza. En este escenario, como el riesgo no necesariamente existiría, se elimina uno de sus elementos básicos y por lo tanto, la validez de su cláusula claims made. Es importante destacar que para la ley se consideran nulos aquellos contratos en que ya haya ocurrido el riesgo, o aquellos que no están expuestos a él (art. 521.2 C.Com., aunque debería propenderse a la nulidad parcial del contrato). Y lo que hace la cláusula claims made al extender retroactivamente la cobertura es, justamente, incorporar un riesgo que ya se ha verificado (porque la intervención o el diagnóstico ya se realizó), o asegurar un riesgo que no ha existido (porque el médico ha actuado diligentemente, sin provocar daños).

Desde un segundo de vista, debe evidenciarse que, aunque los contratantes pueden modificar la llamada "normativa dispositiva" establecida por el legislador, en los seguros esta posibilidad no existe, ya que esta reglamentación, salvo expresamente dispuesto, tiene carácter imperativo y no es modificable a menos que la alteración favorezca al asegurado o al beneficiario (art. 542 C. Com). Bajo esta premisa, podría considerarse inderogable el primer modelo (predispuesto por ley), basado en el sistema de la ocurrencia del daño y no de su reclamación. En este sentido, no resultaría derogable el art. 521.2 C. Com arriba mencionado, ni tampoco se admitiría un sistema -como el de la cláusula claims made-que elimina el periodo de vigencia para la demanda indemnizatoria, ya que si este plazo de prescripción no puede ser reducido (cfr. art. $541 \mathrm{C}$. Com), con mayor razón no puede excluirse. Esta afirmación, surge del hecho que la reforma al contrato de seguro de la Ley n. 20.667/2013, introdujo un plazo de prescripción específico para las acciones emanadas de éste, situación que podría llegar a excluir la validez de cualquier cláusula que elimine dicho término: esto es lo que sucedería con las claims made que huyen de la prescripción y se aplican exclusivamente mediante reclamación hecha dentro del periodo de validez de la póliza. En este sentido, una parte de la literatura sobre el tema considera la ineficacia ${ }^{9}$ o la nulidad absoluta ${ }^{11}$ de estas cláusulas, cada vez que se prevea para el contratante un plazo de prescripción inferior a que tiene la víctima; por ello, el plazo mínimo que debiese ser establecido es de cuatro años, correspondiente al previsto en caso de seguro de vida o aquel de la responsabilidad civil extracontractual (cfr. 541 inc. $4^{\circ}$, 542 C. Com; art. 2332 CC).

Como contrapunto, un argumento en favor de la validez de estas cláusulas podría indicar que éstas no limitan (o expanden) el riesgo contractual, sino que lo que verdaderamente limitan (o expanden), es el contenido del contrato de seguro. De todos modos, aunque se admitiera esta última postura, debe examinarse si, efectivamente, el contratante continúa beneficiado en mayor medida que lo predispuesto por la legislación general (art. 512 C. Com y ss.); y si no fuera así se impondrá, de todos modos, la invalidez de la cláusula.

Ahora bien, normalmente la contratación de una cobertura retroactiva se verifica con un aumento de la prima, lo que, paradójicamente, podría aparejar la desprotección del contrato mismo de seguro. Se trata de una razón más para valorar el eventual carácter abusivo de la cláusula en determinadas circunstancias, como sucede con aquellas en que la retroactividad se extiende solo de manera limitada (cláusula claims made mixta).

\section{Elegir bien es la mejor tutela: la elección de la correcta póliza}

La posible abusividad y consecuente ineficacia de la cláusula claims made destaca interesantes problemáticas que la doctrina ${ }^{9,10}$ ha evidenciado en el caso del consumidor. En tal contexto, correspondería aplicar el art. 16 de la Ley Protección de los Derechos de los Consumidores y sus letras a) y g), que consideran abusiva la limitación de la responsabilidad frente al consumidor o que vayan en contra del principio de buena fe al desequilibrar los derechos y obligaciones de las partes. Sin embargo, en este caso, para demandar la nulidad de la cláusula por estos motivos se necesitará recurrir a las vías judiciales.

Por ello, la mejor tutela para el contratante es actuar antes de elegir la póliza, puesto que luego quedarán limitadas, al encontrarse con un contenido que está ya determinado unilateralmente la compañía de seguro. En efecto, la autonomía contractual de quien celebra la póliza ( $y$, por ende, la posibilidad de eliminar una cláusula claims made) es prácticamente nula. Se trata de un contrato take it or leave it, en que lo único que puede hacer el contratante es elegir (pero no negociar) el tipo de póliza. Por ello - dependiendo de cada situación profesional o actividad desarrollada - debe valorarse si es más conveniente celebrar una póliza tradicional o una que contenga una cláusula claims made.

La posibilidad de acceder a los depósitos de la póliza en la página de la Superintendencia de Valores y Seguros es un punto a favor sobre la transparencia de esta información. Con todo, el contratante muchas veces no poseerá los conocimientos técnicos para 
desentrañar sus diferentes clases y si los tuviera, no sabrá o no podrá saber a cuál depósito de póliza corresponde aquella que ha contratado, información que, a menudo, tampoco viene proporcionada.

En razón de lo anterior, es que, a continuación, se destacan algunas pólizas de seguro de responsabilidad civil médica depositadas en la Superintendencia de Valores y Seguros para proporcionar una panorámica general que muestre ambos escenarios. Esencialmente, deben evidenciarse tres depositadas después de la reforma de 2013: 1) "Póliza de seguro de responsabilidad civil profesional médica" (código POL 120130181) ; 2) "Seguro de responsabilidad civil de médicos" (código POL120131627); 3) "Póliza de seguro de responsabilidad civil profesional médica" (código POL120131523) . La última contiene cláusulas claims made, mientras que las primeras dos se conforman con el primer modelo evidenciado en este escrito (vid. supra n. 4). Se analizan aquí brevemente estas pólizas.

La "Póliza de seguro de responsabilidad civil profesional médica" (código POL 120130181) se califica como póliza clásica (sin cláusula claims made), puesto que en ella se afirma "EI presente seguro ampara la responsabilidad civil del asegurado por daños derivados con ocasión de la prestación de sus servicios durante la vigencia de la póliza". Además, excluye en sus riesgos "A los daños o perjuicios que se hayan producido en una fecha anterior a la de entrada en vigor de la presente póliza, o posterior a la fecha de vencimiento de la misma", riesgos que podrían cubrirse mediante una cláusula claims made.

El "Seguro de responsabilidad civil de médicos" (código POL120131627) también configura una póliza tradicional, puesto que a la hora de describir su cobertura sui art. 4 dispone que "el presente seguro ampara la responsabilidad civil extracontractual del asegurado, generada a consecuencia de actos $u$ omisiones que, teniendo lugar dentro de la vigencia de la póliza, cause muerte, lesiones corporales o daños directos a terceros con ocasión de sus servicios profesionales".

En contra la "Póliza de seguro de responsabilidad civil profesional médica" (código POL120131523) evidencia una tipología de seguro con cláusula claims made, ya que por su art. 19 "La cobertura de esta Póliza es aplicable a las Reclamaciones presentadas por primera vez contra cualquier Asegurado durante la Vigencia de la Póliza o en la Prórroga para Notificaciones en caso en que esta última sea contratada".

Cabe anotar que muchos médicos contrataron su seguro antes de la reforma de 2013, por lo tanto, es posible que se les continúe aplicando un modelo de póliza que actualmente está prohibido por la misma Superintendencia, como es en el caso de la POL 1 91 081. En este caso, la institución hizo un control a posteriori sobre la póliza (y no a priori como en los depósitos), mediante una actividad, fiscalizadora ${ }^{15}$, comprobando que el contenido de la póliza era contrario a la Ley. Dado que dicha prohibición operó con efectos hacia el futuro, quedó abierto el problema en aquellos contratos que se celebraron antes de su prohibición y que todavía se encuentran vigentes. En el caso deesta última póliza debe destacarse que se habían previsto algunas cláusulasalternativas. Por ejemplo, aquella relativa a lamodificación de su art. 1. Al contratar la cláusula alternativa (del art. 1 de la Póliza 191 08, inscrita en el registro de pólizas bajo el código CAL $191082)$ se venía a trasformar el contrato de seguro clásico en otro, que estaba sujeto a una cláusula claims made, puesto que la redacción del artículo 1 conforme a la modificación preveía que: "el presente seguro cubre el riesgo de responsabilidad civil, desde el inicio de la vigencia de la póliza, siempre que el hecho de que derive la responsabilidad cubierta por el seguro, sea reclamado a la compañía a más tardar dentro del año siguiente". Debe tenerse en cuenta la modificación respecto al art. 1 original, que establecía que se cubría la responsabilidad relativas a "daños corporales o patrimoniales que se produzcan durante la vigencia de la póliza".

\section{Obligaciones de medio y de resultado}

Aunque no es objeto principal de este estudio, es útil finalmente hacer una breve referencia a la diferenciación entre las obligaciones de medio y de resultado del médico, por cuanto si bien, en la mayoría de los casos éstas son de la primera tipología, en otros - como ciertas clases de cirugías, procedimientos estandarizados o incluso, actividades desarrolladas por radiólogos - la obligación tendería a acercarse a las de resultado.

En este sentido, cabe destacar que si las pólizas tradicionales excluyen expresamente a las cirugías plásticas y meramente estéticas (POL 120130181; POL 120131523), la Póliza con cláusula claims made (POL 120131627) formalmente descarta todo tipo de responsabilidad de resultado. En su punto 4.6 al referirse a las garantías de resultados, excluye "las obligaciones que conlleven o se deriven del ofrecimiento al PACIENTE, por escrito o no, de la garantía de un determinado resultado de un ACTO MÉDICO específico o las RECLAMACIONES resultantes del incumplimiento de obligaciones de resultado".

Ahora bien, el hecho de que el desarrollo de las actividades de radiología se configura más bien como obligaciones de resultado ${ }^{16}$, podrían hacer peligrar la cobertura del seguro para un radiólogo que contrate esta clase de póliza, aunque la reclamación se haya producido conforme a la cláusula claims made.

\section{Conclusiones}

Se ha intentado aquí dar cuenta de ciertos aspectos de la póliza de responsabilidad civil médica y 
evidenciar ventajas y problemáticas que conllevan las cláusulas de delimitación temporal claims made. Por lo anterior, es oportuno que el médico no sólo cuente con una póliza de seguro válida, sino que estime, dependiendo de su particular actividad profesional, cuál es el modelo que le resulte más conveniente para su cobertura. Así que, aunque quede ya protegido, en parte, por una póliza gremial, debe considerar si ésta puede complementarse con una adicional que cubra eventuales responsabilidades particulares de la especialidad que el médico en concreto desarrolla. En todo esto juega un rol fundamental la información, que puede ser proporcionada por la propia compañía de seguro, o por la Superintendencia de Valores y Seguros mediante el acceso al depósito de las pólizas en su página web. Por su parte, el mismo contratante tiene un deber de informarse, el que será directamente proporcional a su nivel de profesionalización.

En este contexto, las asociaciones gremiales podrían jugar un rol importante en favor de sus miembros, contribuyendo con la entrega de información que favorezca el pleno conocimiento de este panorama. Sería aconsejable, además, ofrecer al afiliado la posibilidad de elegir entre la celebración de una póliza de responsabilidad profesional tradicional u otra que contenga las cláusulas claims made.

Hace algunas décadas habría sido inimaginable la evolución que ha experimentado la responsabilidad médica, al punto de considerarse hoy como una rama específica y con una operatividad especial dentro de la responsabilidad civil.

Este desarrollo, sumado a la oferta de nuevas pólizas de seguro, aconseja no dejar fuera ninguna temática, por muy específica y técnica que ésta sea. El avance de los seguros hoy podría hacer reales fenómenos que, de momento, son minoritarios o que existen, pero son escasamente divulgados. Y si bien las cláusulas de delimitación temporal son poco conocidas, esta problemática requiere ser abordada, pues se trata de un fenómeno real, que está presente en las actuales pólizas y del cual depende la efectiva cobertura que puede recibir un médico por parte de su compañía de seguros.

\section{Referencias}

1. Montoya $D$, Rosmanich $P$, Velásquez V, López J. Aspectos médicos y legales de las querellas por responsabilidad médica. Rev Med Chile 1993; 121: 679-685.

2. Acosta V. Responsabilidad civil médica en Chile. Rev Chil Radiol 1997; 3: 5-17.

3. Etcheberry A. Responsabilidad legal del médico. Primeras Jornadas de Ética Médica. Colegio Médico de Chile. Santiago. 1984; 39-60.

4. Barros E. Tratado de responsabilidad extracontractual. Santiago: Jurídica, 2006; en particular 656-694.

5. Corral H. Lecciones de responsabilidad civil extra- contractual. Santiago: Jurídica, 2013; en particular 283-291.

6. Pizarro C. Mal praxis en el equipo médico. Rev. Med. Chile 2011; 139: 667-671.

7. Pizarro C. Responsabilidad profesional médica: Diagnóstico y perspectivas. Rev. Med. Chile 2008; 136: 539-554.

8. Achurra J, Arellano S, Contreras O, Salinas G, Sateler R. El futuro del seguro de responsabilidad civil. Derecho de Seguros. Escritos de Juan Achurra Larraín. tomo 2, Santiago, Universidad de los 9.Andes, 2005; 131-183, en particular 164-183.

9. Isler E. Acerca de la validez de las cláusulas claims made. Escobar M. (ed.), Estudios de derecho comercial. VII ${ }^{a}$ Jornadas chilenas de derecho comercial 2016. Santiago: Rubicon 2017; 531-546.

10. Lagos O. “art. 532”, en Ríos R. (Dir.), Schiele C. (ed.), El contrato de seguro. Comentarios al título VIII, libro II, de código de comercio, Santiago: Thomson Reuters 2015; 374-378.

11. Barrientos M. "art. 541", en Ríos R. (Dir.), Schiele C. (ed.), El contrato de seguro. Comentarios al título VIII, libro II, de código de comercio, Santiago: Thomson Reuters 2015; 456-490.

12. Dulia T, César M. Prevención de riesgo en radiología: el error y el radiólogo. Revista Chilena de Radiología 2002; 3: 135-140.

13. Ferrante A. La incorporación de la cláusula claims made en el contrato de seguro de responsabilidad civil según la nueva visión italiana. Revista chilena de Derecho Privado 2016; 27: 229-234.

14. Contreras O. Derecho de Seguros, $2^{\underline{a}}$ ed., Santiago: Legal Publishing, 2014; en particular 196.

15. Ríos R. "art. 542". Ríos R. (Dir.), Schiele C. (ed.), El contrato de seguro. Comentarios al título VIII, libro II, de código de comercio, Santiago: Thomson Reuters 2015; 461-473.

16. Manrique Jl. Patología y radiología. Obligación de medio o de resultado. Rev. Médico Legal 2007; 4: 20-23.

i. Véase por ejemplo la Corte Suprema 10/03/2016, rol n. 21373-2015.

ii. Hay que matizar que, en virtud del tercer inciso del art. 541 CCom, en el caso de seguro de vida el beneficiario posee un particular plazo de prescipción, que será de cuatro años y se contará "desde que conoce la existencia de su derecho, pero en ningún caso excederá de diez años desde el siniestro".

iii. Aunque existen varias tipologías de cláusulas claims made, esencialmente se presentan estas dos. Frente al escaso tratamiento de esta cláusula en la doctrina chilena, parece oportuno aportar una definición que ha entregado la Suprema Corte italiana, que brilla por su claridad: "A pesar de que en la praxis mercantil existan múltiples tipologías de cláusulas claims made el esquema puede acotarse a dos grandes categorías: a) las cláusulas llamadas mixtas o impuras, que prevén la operatividad del contrato de seguro sólo cuando tanto el hecho ilícito como la solicitud de indemnización se verifiquen durante la eficacia del contrato o con efecto retroactivo de la garantía, en determinados casos (...) con inclusión de conductas realizadas anteriormente (normalmente dos o tres años antes de la celebración 
del contrato); b) cláusulas llamadas puras, destinadas a garantizar todo tipo de solicitud de daños y perjuicios cursadas por la víctima al asegurado y que éste remitió al seguro durante el período de vigencia de la póliza, sin importar la fecha de hecho ilícito". Corte di Cassazione a sezioni unite n. $9140 / 2016$, de 6 de mayo.Corriere giuridico; 2016; 7; 921; Danno e responsabilità, 10, 2016, 926-935;Contratti; 8-9; 753-758. Sobre esta sentencia en idioma castellano se remite a (13). 